ANNALES

POLONICI MATHEMATICI

XLIX (1989)

\title{
On an integral inequality for piecewise continuous functions
}

\author{
by P. S. Simeonov and D. D. Bainov (Plovdiv)
}

\begin{abstract}
In the paper, the decision problem of integral inequalities of the type BellmanBihari is discussed. The obtained results can successfully be used in the fundamental, qualitative and asymptotic theory of the differential systems with impulse effect.
\end{abstract}

1. Introduction. Systems with impulse effect [2]-[4] are characterized by the fact that their solutions have first order discontinuities at the moments of impulse effect where they are left continuous.

The qualitative research of similar systems often involves a new class of integral inequalities where the estimated function $u(t)$ is piecewise continuous and its jump at the discontinuity points $t_{k}$ depends on the value $u\left(t_{k}\right)$ (or $\left.u\left(t_{k}-0\right)\right)$. The analogue of the Gronwall-Bellman inequality [4] is an example of a similar inequality,

$$
u(t) \leqslant c+\int_{a}^{t} p(s) u(s) d s+\sum_{a<t_{i}<i} \beta_{i} u\left(t_{i}-0\right) .
$$

The present paper considers the problem for the solvability of an integral inequality containing a large class of inequalities, often met in the study of systems with impulse effect [4]-[8].

2. Preliminary notes. Let $\boldsymbol{R}^{n}$ be the $n$-dimensional Euclidean space with norm $|\cdot|$ and let $B_{r}=\left\{x \in \boldsymbol{R}^{n}:|x|<r\right\}$. The space $\boldsymbol{R}^{n}$ will be considered to be semiordered in the following sense: we will write $u \leqslant v$ if $u_{i} \leqslant v_{i}, i=1, \ldots, n$. If $G \subset \boldsymbol{R}^{n}$, then the function $\psi: G \rightarrow \boldsymbol{R}^{n}$ will be called nondecreasing in $G$ if $u \leqslant v, u \in G, v \in G$ implies that $\psi(u) \leqslant \psi(v)$.

Let the function $K(t, s, u)$ be defined for $\alpha \leqslant s \leqslant t<\beta \leqslant \infty, u \in B_{c}$ $(0<c \leqslant \infty)$.

We will say that conditions (K) hold provided the following conditions are satisfied:

$\left(K_{1}\right)$ The function $K(t, s, u)$ is continuous with respect to $u$ for all $t$ and almost all $s$, and it is measurable with respect to $s$ for all $t$ and $u$. 
$\left(\mathrm{K}_{2}\right) \quad$ For all $t$ and for almost all $s$, the function $K(t, s, u)$ is nondecreasing in $B_{c}$.

$\left(\mathrm{K}_{3}\right) \quad$ For each $d \in(0, c)$, functions $\mu_{d}(t, s)$ and $v_{d}(\tau, t, s)(\alpha \leqslant s \leqslant t \leqslant \tau<\beta)$ exist, summable with respect to $s$ in $[\alpha, t]$, and such that

$$
\sup _{|u| \leqslant d}|K(t, s, u)| \leqslant \mu_{d}(t, s), \quad \sup _{|u| \leqslant d}|K(\tau, s, u)-K(t, s, u)| \leqslant v_{d}(\tau, t, s) .
$$

$$
\lim _{\tau-t \rightarrow 0_{+}} \int_{i}^{\tau} \mu_{d}(\tau, s) d s=0 \quad \text { for a fixed } t \text { or } \tau \text {. }
$$

$$
\lim _{\tau-t \rightarrow 0_{+}} \int_{a}^{t} v_{d}(\tau, t, s) d s=0 \quad \text { for a fixed } t \text { or } \tau
$$

$\left(\mathbf{K}_{6}\right)$

$$
\lim _{\tau \rightarrow t \rightarrow 0_{+}} \int_{\alpha}^{i} v_{d}(\tau, t, s) d s=0 \quad \text { for a fixed } t \neq \tau_{i} \text { or } \tau \neq \tau_{i},
$$

where the sequence $\left\{\tau_{i}\right\}$ is such that $\alpha<\tau_{1}<\ldots<\tau_{i}<\ldots, \lim _{i \rightarrow \infty} \tau_{i}$ $\geqslant \beta$.

3. Main results. The basic results are grounded on the following lemmas:

LEMMA 1 [1]. Let the following conditions be fulfilled:

1. Conditions $\left(\mathrm{K}_{1}\right)-\left(\mathrm{K}_{5}\right)$ hold.

2. The function $\varphi:[\alpha, \beta) \rightarrow \boldsymbol{R}^{n}$ is continuous and $|\varphi(\alpha)|<c$.

3. The function $v:[\alpha, \beta) \rightarrow B_{c}$ is the maximal (minimal) noncontinuable solution of the equation

$$
v(t)=\varphi(t)+\int_{\alpha}^{t} K(t, s, v(s)) d s, \quad t \in[\alpha, \beta) .
$$

4. The function $u:[\alpha, \beta) \rightarrow B_{c}$ is continuous and satisfies the integral inequality

$$
\Delta(t) \equiv u(t)-\varphi(t)-\int_{\alpha}^{t} K(t, s, u(s)) d s \leqslant 0 \quad(0 \leqslant \Delta(t)), t \in[\alpha, \beta) .
$$

Then for $t \in[\alpha, \beta)$,

$$
u(t) \leqslant v(t) \quad(v(t) \leqslant u(t)) .
$$

LEMMA 2. Let the following conditions be fulfilled:

1. Conditions $\left(\mathrm{K}_{1}\right)-\left(\mathrm{K}_{4}\right)$ and $\left(\mathrm{K}_{6}\right)$ hold.

2. The function $\varphi:[\alpha, \beta) \rightarrow \boldsymbol{R}^{n}$ is piecewise continuous and $|\varphi(\alpha)|<c$. 
3. The function $v:[\alpha, \beta) \rightarrow \boldsymbol{R}^{n}$ is the maximal (minimal) solution of the equation

$$
v(t)=\varphi(t)+\int_{\alpha}^{t} K(t, s, v(s)) d s, \quad t \in[\alpha, \beta)
$$

4. The function $u:[\alpha, \beta) \rightarrow \boldsymbol{R}^{n}$ is piecewise continuous and satisfies the integral inequality

$$
\Delta(t) \equiv u(t)-\varphi(t)-\int_{\alpha}^{t} K(t, s, u(s)) d s \leqslant 0 \quad(0 \leqslant \Delta(t)), \quad t \in[\alpha, \beta) .
$$

5. For each $\gamma \in(\alpha, \beta)$ the inequalities

$$
\sup _{t \in[a, \gamma]}|v(t)|<c, \quad \sup _{t \in[a, \gamma]}|u(t)|<c
$$

are satisfied.

6. The discontinuity points of the functions $\varphi, v$ and $u$ are contained in the sequence $\left\{\tau_{i}\right\}$.

Then for $t \in[\alpha, \beta)$

$$
u(t) \leqslant v(t)(v(t) \leqslant u(t)) .
$$

Proof. In virtue of Lemma 1, inequality (2) holds for $t \in\left[\alpha, \tau_{1}\right) \cap[\alpha, \beta)$. Assume that for some $i=1,2, \ldots$ and for every $t \in\left[\alpha, \tau_{i}\right) \cap[\alpha, \beta)$

$$
u(t) \leqslant v(t) \text {. }
$$

Since the function $K(t, s, u)$ is nondecreasing with respect to $u$, for $t \in\left[\tau_{i}, \tau_{i+1}\right) \cap[\alpha, \beta)$ we have

$$
u\left(\tau_{i}\right) \leqslant \varphi\left(\tau_{i}\right)+\int_{a}^{\tau_{i}} K\left(\tau_{i}, s, u(s)\right) d s \leqslant v\left(\tau_{i}\right)
$$

and

$$
w(t) \leqslant \Phi_{i}(t)+\int_{\tau_{i}}^{t} K(t, s, w(s)) d s, \quad t \in\left[\tau_{i}, \tau_{i+1}\right) \cap[\alpha, \beta),
$$

where

$$
\begin{gathered}
w(t)= \begin{cases}u(t), & t \in\left(\tau_{i}, \tau_{i+1}\right), \\
u\left(\tau_{i}+0\right), & t=\tau_{i},\end{cases} \\
\Phi_{i}(t)=\varphi(t)+\int_{\alpha}^{\tau_{i}} K(t, s, v(s)) d s, \quad t \in\left(\tau_{i}, \tau_{i+1}\right) \cap[\alpha, \beta),
\end{gathered}
$$

$\Phi_{i}\left(\tau_{i}\right)=\Phi_{i}\left(\tau_{i}+0\right)$. 
(5), (6) and conditions 1 and 5 of Lemma 2 imply that the function $\Phi_{i}(t)$ is continuous on $\left[\tau_{i}, \tau_{i+1}\right) \cap[\alpha, \beta)$ and

$$
\begin{aligned}
\left|\Phi_{i}\left(\tau_{i}\right)\right| & =\left|\Phi_{i}\left(\tau_{i}+0\right)\right| \\
& =\left|\varphi\left(\tau_{i}+0\right)+\lim _{t \rightarrow \tau_{i}+0} \int_{a}^{\tau_{i}} K(t, s, v(s)) d s\right| \\
& =\left|\varphi\left(\tau_{i}+0\right)+\lim _{t \rightarrow \tau_{i}+0} \int_{\alpha}^{t} K(t, s, v(s)) d s\right| \\
& =\left|v\left(\tau_{i}+0\right)\right|<c .
\end{aligned}
$$

Then, in view of Lemma 1,

$$
u(t)=w(t) \leqslant \xi(t), \quad t \in\left(\tau_{i}, \tau_{i+1}\right) \cap[\alpha, \beta),
$$

where $\xi(t)$ is the maximal solution of the equation

$$
\xi(t)=\Phi_{i}(t)+\int_{i_{i}}^{t} K(t, s, \xi(s)) d s, \quad t \in\left[\tau_{i}, \tau_{i+1}\right) \cap[\alpha, \beta) .
$$

However, (5) and (8) imply that the function

$$
\alpha(t)= \begin{cases}v(t), & t \in\left[\alpha, \tau_{i}\right] \cap[\alpha, \beta), \\ \xi(t), & t \in\left(\tau_{i}, \tau_{i+1}\right) \cap[\alpha, \beta)\end{cases}
$$

is a solution of (1) in the interval $\left[\alpha, \tau_{i+1}\right) \cap[\alpha, \beta)$. Then, (3), (4) and (7) yield that

$$
u(t) \leqslant \alpha(t) \leqslant v(t), \quad t \in\left[\alpha, \tau_{i+1}\right) \cap[\alpha, \beta) .
$$

Hence, inequality (3) is verified for each interval $\left[\alpha, \tau_{i}\right) \cap[\alpha, \beta), i$ $=1,2, \ldots$, i.e., inequality (2) holds for $t \in[\alpha, \beta)$.

THEOREM 1. Let the following conditions be fulfilled:

1. Conditions $\left(\mathrm{K}_{1}\right)-\left(\mathrm{K}_{4}\right)$ and $\left(\mathrm{K}_{6}\right)$ hold.

2. The sequence $\left\{t_{i}\right\}_{i=1}^{\infty}$ is such that

$$
\alpha<t_{1}<\ldots<t_{i}<\ldots, \quad \lim _{i \rightarrow \infty} t_{i} \geqslant \beta .
$$

3. The function $\varphi:[\alpha, \beta) \rightarrow \boldsymbol{R}^{n}$ is piecewise continuous and $|\varphi(\alpha)|<c$.

4. The functions $\psi_{i}(t, s, u), i=1,2, \ldots$, are defined for $t, s \in[\alpha, \beta)$, $u \in B_{c}$; they have values in $\boldsymbol{R}^{n}$; they are nondecreasing in $B_{c}$ for fixed $t$ and $s$; they are piecewise continuous with respect to $t$ for fixed $s$ and $u$.

5. The function $v:[\alpha, \beta) \rightarrow \boldsymbol{R}^{n}$ is the maximal (minimal) noncontinuable solution of the equation

$$
v(t)=\varphi(t)+\int_{\alpha}^{t} K(t, s, v(s)) d s+\sum_{\alpha<t_{i}<t} \psi_{i}\left(t, t_{i}, v\left(t_{i}\right)\right), \quad t \in[\alpha, \beta) .
$$


6. The function $u:[\alpha, \beta) \rightarrow \boldsymbol{R}^{n}$ is piecewise continuous and satisfies the inequality

$$
\begin{aligned}
\Delta(t) \equiv u(t)-\varphi(t)-\int_{a}^{t} K(t, s, u(s)) d s-\sum_{\alpha<t_{i}<t} \psi_{i}\left(t, t_{i}, u\left(t_{i}\right)\right) \leqslant 0, & 0 \leqslant \Delta(t), t \in[x, \beta) .
\end{aligned}
$$

7. For every $\gamma \in(\alpha, \beta)$ the inequalities

$$
\sup _{t \in[\alpha, y]}|v(t)|<c, \quad \sup _{t \in[x, y]}|u(t)|<c
$$

hold.

8. The points $\left\{t_{i}\right\}$ and the discontinuity points of the functions $\varphi, u, v$ and $\psi_{i}, i=1,2, \ldots$, are contained in the sequence $\left\{\tau_{i}\right\}$.

Then, for $t \in[\alpha, \beta)$,

$$
u(t) \leqslant v(t) \quad(v(t) \leqslant u(t)) .
$$

The verification of inequality (11) over the interval $\left[\alpha, \tau_{i}\right) \cap[\alpha, \beta), i$ $=1,2, \ldots$, is proved by induction with respect to $i$, employing the same arguments as in the proof of Lemma 2 with the only difference that the function

$$
\begin{aligned}
& \Phi_{i}(t)=\varphi(t)+\int_{\alpha}^{\tau_{i}} K(t, s, v(s)) d s+\sum_{\alpha<\tau_{k}<\tau_{i}} \psi_{k}\left(t, t_{k}, v\left(t_{k}\right)\right), \\
& t \in\left(\tau_{i}, \tau_{i+1}\right) \cap[\alpha, \beta)
\end{aligned}
$$

is used and Lemma 2 is applied instead of Lemma 1.

Remark 1. Theorem 1 still holds if in one and the same summands of (9) and (10) the values $v\left(t_{i}\right)$ and $u\left(t_{i}\right)$ are replaced by $v\left(t_{i}-0\right)$ and $u\left(t_{i}-0\right)$.

Remark 2. Note that the discontinuity points of the function $u(t)$ may not coincide with the "jump" points $\left\{t_{i}\right\}$. This is of particular importance in view of the applications in the theory of stability of systems with impulse effect in the cases when the moments of impulse effect of the considered system are not fixed [7].

Corollary 1. Let for $\alpha \leqslant t<\beta \leqslant \infty$

$$
u(t) \leqslant c \prod_{\alpha<t_{i}<t} p_{i}+\int_{a}^{t} \prod_{s \leqslant t_{i}<t} p_{i} p(s) u^{m}(s) d s+\sum_{\alpha<t_{i}<t}\left(\prod_{t_{i} \leqslant t_{j}<t} p_{j}\right) \beta_{i} u\left(t_{i}\right),
$$

where $p_{i}>0, \beta_{i} \geqslant 0, c \geqslant 0, p:[\alpha, \beta) \rightarrow[0, \infty)$ is continuous, $u:[\alpha, \beta)$ $\rightarrow[0, \infty)$ is piecewise continuous, $m>0$. 
Then, for $m \neq 1$

$$
u(t) \leqslant c \prod_{a<t_{i}<t} p_{i}\left(1+\beta_{i}\right)\left[1-(m-1) \int_{x}^{1}\left(c \prod_{x<t_{i}<s} p_{i}\left(1+\beta_{i}\right)\right)^{m-1} p(s) d s\right]^{1 /(1-m)}
$$

for all $t \geqslant \alpha$ for which

$$
(m-1) \prod_{a}^{t}\left(c \prod_{a<t_{i}<s} p_{i}\left(1+\beta_{i}\right)\right)^{m-1} p(s) d s<1
$$

while for $m=1$

$$
u(t) \leqslant c \prod_{x<t_{i}<1} p_{i}\left(1+\beta_{i}\right) \exp \left(\int_{\alpha}^{t} p(s) d s\right) \text { for } t \geqslant \alpha .
$$

Remark 3. For $p_{i} \equiv 1, m=1$, the result of Corollary 1 coincides with the result of Lemma 2 [4] and is actually a generalization of the GronwallBellman Lemma.

\section{References}

[1] N. V. Azbelev, Z. B. Tsaljuk, On integral inequalities, 1 (in Russian). Math. Sb. 56 (98) (1962), 325-342.

[2] V. D. Mil'man, A. D. Myshkis, On the stability of motion in the presence of impulses (in Russian), Sib. Math. Z. 1 (1960), 233-237.

[3] S. G. Pandit, S. G. Deo, Differential systems intolving impulses, Springer-Verlag, BerlinHeidelberg-New York 1982.

[4] A. M. Samoilenko, N. A. Perestjuk, Stability of the solutions of differential equations with impulse effect (in Russian). Differential Equations 11 (1977), 1981-1992.

[5] P. S. Simeonov, D. D. Bainov, Stability in linear approximation of systems with impulse effect, Rend. Mat. Univers Politecn. Torino 43 (1985), 303-321.

[6] -., -, Asymptotic stability of the solutions of integro-differential equations with impulse effect (to appear).

[?] -, -. Stability under persistent disturbances for systems with impulse effect (to appear).

[8] -, -, Asymprotic equivalence of two systems of differential equations with impulse effect, Systems and Control Letlers 3 (1983), 297-301. 